\title{
Fast Streaking Artifact Reduction in CT Using Constrained Optimization in Metal Masks
}

\author{
Jonas August and Takeo Kanade \\ Robotics Institute, Carnegie Mellon University, Pittsburgh, PA
}

\begin{abstract}
Here we accelerate computations that reduce CT metal artifacts by observing that metal projects to only a fraction of the X-ray detectors in each projection, and thus computations should focus on these metal "mask" regions. We propose that the penalized maximum likelihood optimization method for artifact reduction needs to be solved only within the metal mask, using the remaining non-mask regions as a constraint; we show that our approach leads to a 10x speedup.
\end{abstract}

Modern methods for reducing the streaking artifacts caused by metal in CT images (Fig. 1, top left) are based on solving an optimization problem where the unknown is the entire image [3]. Unfortunately, such solutions require computations far greater than the cause: the metal, which is typically a small area in the image. X-rays that project through metal are highly attenuated and thus have greater photon noise; during reconstruction the streaks form due to the backprojection of this noisy metal "mask" region. Projection completion methods suppress streaks by interpolating across such noisy metal portions of each projection before reconstruction; however, such ad-hoc techniques suffer from sensitivity to estimates of the metal mask. In this paper, we combine both ideas to accelerate artifact reduction by restricting the optimization to the metal mask.

In the penalized maximum likelihood method [2], one seeks that image $f$ that minimizes the left hand side of

$$
\|g-\mathcal{R} f\|_{\mathcal{W}}^{2}+\|\nabla f\|^{2}=\|g-h\|_{\mathcal{W}}^{2}+\frac{1}{4 \pi}\left\|\mathcal{I}^{-3 / 2} h\right\|^{2},
$$

given only the projection data $g$, where $\mathcal{R}$ is the Radon transform (which takes line integrals) and $\|\cdot\|_{\mathcal{W}}$ is a weighted norm with a diagonal weight matrix $\mathcal{W}$ which is smaller in the metal regions of each projection. The first term ensures closeness to the data primarily in lower-attenuation regions, while the second enforces smoothness by penalizing gradients in $f$. Equivalently [1], one can instead minimize the right hand side of (1) with respect to $h=\mathcal{R} f$, where $\mathcal{I}^{-1}$ is the ramp filter used in filtered backprojection $\left(\mathcal{R}^{-1} \propto \mathcal{R}^{T} \mathcal{I}^{-1}\right.$, where $\mathcal{R}^{T}$ is the backprojection operator). The advantage of optimizing over $h$ is that the projections (one per angle) can be optimized separately without loss, since the ramp acts only along each projection. The final image is then $f=\mathcal{R}^{-1} h$.

Given a mask region $\mathcal{M}$, we accelerate the optimization over $h$ by adding the equality constraint that $h=g$ outside of $\mathcal{M}$, where the data is less noisy. This gives rise to a much smaller set of equations for $h$ within the noisy region $\mathcal{M}$ only, solved using a direct sparse solver in Matlab. The solution to the unconstrained optimization problem for all projections for the image in Fig. 1 took 439 seconds 
on a $2.4 \mathrm{GHz}$ Xeon, but only 42 seconds using the mask method, but the two solutions produce comparable images. These ideas also apply to regularized $3 \mathrm{~d}$ cone-beam reconstruction, where we expect even greater speedup.
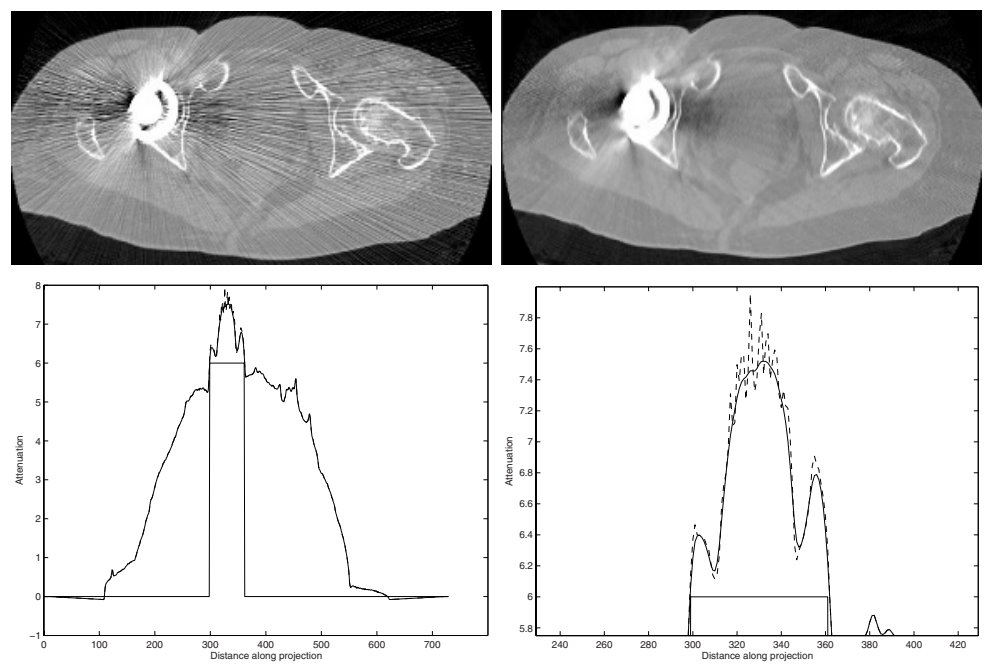

Fig. 1. Streaking artifacts that radiate from a metal hip implant in this CT image (top left) can be removed (top right) by solving optimization problem (1) along each projection (bottom left, with zoom of peak in bottom right). The dashed rough contour depicts the noisy CT projection data $g$ corresponding to a single orientation from the sinogram (not shown); the solid smoother contour is the solution $h$ to the optimization problem. These curves differ mostly near the peak where the noise is greatest. This noise is responsible for the streak artifacts produced by filtered backprojection. Here we propose a method to accelerate artifact reduction by solving the optimization problem only within the metal portion or "mask" along each projection where the data is most noisy; the mask is indicated with the rectangular impulse, and represents a fraction of the entire projection, since metal objects in $\mathrm{CT}$ tend to be small relative to the body. Thus the computation scales with the size of the metal, unlike other optimization approaches that solve for the entire image [4. Note we do not require a precise mask, since it only limits the domain of smoothing. Here the mask is simply the data thresholded at an attenuation of 6 ; the constraint prevents mask-border effects.

\section{References}

1. J. August. Decoupling the equations of regularized tomography. In International Symposium on Biomedical Imaging, pages 653-656, 2002.

2. J. A. Fessler and S. D. Booth. Conjugate-gradient proconditioning methods for shift-variant pet image reconstruction. IEEE Trans. on Image Proc., 8(5):688-699, 1999.

3. B. D. Man, J. Nuyts, P. Dupont, G. Marchal, and P. Suetens. Reduction of metal streak artifacts in x-ray computed tomography using a transmission maximum a posteriori algorithm. IEEE Trans. on Nuclear Science, 47(3):977-981, 2000.

4. G. Wang, T. Frei, and M. W. Vannier. Fast iterative algorithm for metal artifact reduction in x-ray ct. Acad. Radiol., 7:607-614, 2000. 